\title{
Physical activity level is impaired and diet dependent in preterm newborn pigs
}

\author{
Muqing Cao' ${ }^{1}$ Anders Daniel Andersen ${ }^{2,3}$, Chris Van Ginneken ${ }^{4}$, René Liang Shen ${ }^{2,3}$, Stine Ostenfeldt Petersen ${ }^{2,3}$, \\ Thomas Thymann ${ }^{2,3}$, Jin Jing ${ }^{1}$ and Per Torp Sangild ${ }^{2,3,5}$
}

BACKGROUND: Preterm infants show delayed development of motor function after birth. This may relate to functional immaturity of many organs, including the gut and brain. Using pigs as model for preterm infants, we hypothesized that early initiation of enteral feeding stimulates both gut growth and neonatal physical activity.

METHODS: In experiment 1, preterm and term pigs were fed parenteral nutrition (PN) or PN plus bovine colostrum (BC, $16-64 \mathrm{ml} / \mathrm{kg} / \mathrm{d}$ enterally) for $5 \mathrm{~d}$. In experiment 2, preterm pigs were fed $\mathrm{PN}+\mathrm{BC}$ or PN+formula for $5 \mathrm{~d}$. In experiment 3, preterm pigs were fed $B C$, formula, or human milk (HM) for $10 \mathrm{~d}$. Incubator home cage activity (HCA) was quantified by continuous camera recordings.

RESULTS: Preterm birth was associated with reduced intestinal weight and HCA (experiment 1), and BC or formula supplementation increased intestinal weights and HCA (experiments 1+2). Enteral BC and HM feeding increased HCA, intestinal weights, and necrotizing enteritis resistance, relative to formula (experiment 3).

CONCLUSION: Preterm pigs show decreased physical activity, and the first enteral feeds diet dependently stimulate both gut growth and physical activity. The effects may arise from maturation of digestive, metabolic, and neurological functions, including gut serotonin production, by the first enteral feeds and milk bioactive factors.

$\mathbf{P}$ reterm infants are a high-risk patient population with a variety of developmental complications, including delayed neurodevelopment and a high incidence of brain insults. Even when newborn preterm infants show no clinically observable brain defects, it is common to observe that such infants exhibit slower postnatal development of motor skills, compared with term infants. Even at term-corrected age, the brains in preterm infants may show compromised gray matter volume and altered developmental trajectory (1), probably explaining delayed neonatal arousal $(2,3)$ and immature neuromuscular function and brain electroencephalography (4). In more longterm studies, preterm infants have shown reduced postural complexity (5) and lowered motor function (6), potentially persisting until school age (7), or even into adulthood (8). Thus, early impairment of physical locomotion in preterm infants may have long-term consequences.

There is rapidly increasing evidence of functional links among the gastrointestinal tract (GIT), the diet, and the brain, especially in early life (9). Growth rate and nutrient intakes in early life may affect later neuromuscular and cognitive outcomes, but the mechanisms are largely unknown (10). Much focus has been devoted to the possible role of the lipid fraction of milk, but firm evidence for diet effects are lacking, not only in infants (11) but also in various animal models (12). In addition to the direct effects of nutrients and dietary factors on neurodevelopmental endpoints, indirect effects may arise from links between the early diet on gut growth, enteric nervous system, signaling molecules like serotonin (5-HT), and/ or gut microbiota. Studies in mice show that changes in the gut microbiota after birth, in parallel with the first enteral feeding, lead to an anxiety-depression-like behavior (13) and other behavioral changes (14). Whether such effects are mediated by changes in gut physiology and microbiology, resulting in metabolic, endocrine, or neuronal effects on the brain and central nervous system (CNS), are unknown.

Preterm very-low-birth-weight infants have difficulties in tolerating enteral feeding just after birth due to a series of gut structural and functional immaturities that include an immature enteric nervous system, as demonstrated in preterm pigs fed total parenteral nutrition (TPN) or enterally $(15,16)$. On the other hand, it is important to start enteral feeding as early as possible because even small amounts of enteral feeds may support gut growth, function, and bacterial colonization. Because enteral feeding, given as an adjunct to PN (e.g., "minimal enteral feeding" (MEN)), may facilitate an earlier transition to later full enteral feeding (i.e., $>150 \mathrm{ml} / \mathrm{kg} / \mathrm{d}$ ), MEN may be important to reduce the postnatal growth deficit experienced

'Department of Maternal and Child Health, Faculty of Public Health, Sun Yat-sen University, Guangzhou, China; ${ }^{2}$ Clinical and Experimental Nutrition, Department of Nutrition,

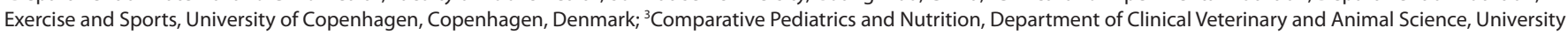
of Copenhagen, Copenhagen, Denmark; ${ }^{4}$ Department of Veterinary Sciences, Faculty of Pharmaceutical, Biomedical \& Veterinary Sciences, University of Antwerp, Antwerp, Belgium; ${ }^{5}$ Department of Pediatrics and Adolescent Medicine, Copenhagen University Hospital (Rigshospitalet), Copenhagen, Denmark. Correspondence: Per Torp Sangild (psa@nexs.ku.dk) 
by many very-low-birth-weight infants (17). This deficit may further compromise maturation of many organs, including the brain. Conversely, a slow transition to enteral feeding, using an optimal milk diet such as human milk (HM, either mother's own milk or donor milk) or bovine colostrum (BC), is required to avoid feeding intolerance and serious gut complications, such as necrotizing enteritis (NEC) in both infants and animal models (18-20). Incidence of NEC generally increases with rapid progression of enteral feeding, especially using infant formula (IF), although the optimal feeding procedure remains debated.

On this background, we hypothesized that (i) preterm birth is associated with impaired physical activity and gut growth, (ii) MEN feeding improves physical activity and gut growth, and (iii) neonatal physical activity is diet dependent. We used our well-established preterm pig model to investigate how different diet and feeding patterns would affect neonatal arousal, physical activity, and gut growth after preterm and term birth. In this initial series of experiments, we documented the proposed associations among early enteral feeding, gut growth, and overall physical activity and made preliminary investigations into mechanisms involving serotonin. In experiment 1 , both preterm and term piglets received TPN or PN+MEN for $5 \mathrm{~d}$, whereas experiment 2 compared the neonatal effects of two MEN diets (BC, IF). In experiment 3 , the neonatal physical activity and gut weights were recorded after feeding a slightly higher enteral dose of three diets (BC, HM, and IF) for $10 \mathrm{~d}$ (Table 1).

\section{RESULTS}

\section{Relative Gut Weight, NEC Score, and Body Weight Gain}

In experiment 1 , intestinal weight to body weight at $5 \mathrm{~d}$ of age was significantly higher in term vs. preterm pigs $(P<$ 0.01 ; Table 2). Conversely, term pigs had lower relative brain weights, compared with preterm pigs. For both delivery ages, MEN-BC pigs showed increased intestinal weights, relative to TPN $(P<0.01)$, with no effect on brain weight. By day 5 , none of the pigs showed any clinical evidence of NEC lesions in their small intestine, but one preterm and one term MEN-BC pig showed mild lesions (NEC scores 3-4) in either the stomach or colon regions, resulting in NEC classification (Table 2).
In experiment 2, TPN pigs had higher body weight gain, but lower intestinal weights, than pigs fed MEN levels of colostrum or formula $(P<0.05$; Table 2$)$, with no effects on brain weights. Growth rate tended to be lower for MEN-IF, relative to MEN-BC pigs $(P=0.08)$, and these former pigs also showed elevated colon weight, compared with the other groups $(P<0.05$; Table 2$)$. This is explained by the predominant location of NEC lesions for this group to the colon region, resulting in accumulation of fluid in edematous and inflamed tissue. Colon NEC score was $3.1 \pm 0.5$ in the MEN-IF pigs vs. $1.5 \pm 0.3$ and $1.0 \pm 0.0$ in the TPN and MEN-BC groups, respectively (TPN vs. MEN-IF, $P=0.08$; TPN vs. $M E N-B C, P=0.15$; MEN-IF vs. MEN-BC, $P=0.002)$. A significant correlation was found between colon NEC score and relative colon weight $(P$ $<0.01 ; r=0.60$ for MEN-IF pigs). Despite these observations at tissue collection, none of the MEN-IF pigs showed any of the typical clinical signs of discomfort and NEC in the days before tissue collection (e.g., abdominal distension, lethargy, discoloration, respiratory distress, and bloody stools), suggesting that the progression of NEC was still at a relatively early stage with a low severity.

In experiment 3, EN-HM pigs had lowered weights of the small intestine and colon, relative to the EN-BC and EN-IF pigs $(P<0.05$; Table 2$)$ at tissue collection on day 10 (e.g., $4 \mathrm{~d}$ after stopping the activity recordings on day 6$)$. At this time, NEC incidence was relatively high in all pigs $(\sim 60 \%$ across all three diet groups). In both the stomach and colon, average NEC lesion score was higher in the EN-IF group, relative to the other two groups which were similar (1.9 vs. 1.1 and 2.6 vs. 1.8 , respectively, both $P<0.05$ ). The tendency to a lower NEC incidence and severity in the EN-HM group, relative to the two other groups, was reflected mainly by a lowered score in the small intestine ( 1.5 vs. $2.2 ; P<0.05$ ). There was no effect of diet on relative brain weights.

\section{Neonatal Arousal, Time to First Stand, and Time to First Walk}

Preterm pigs required significantly more time to achieve basic motor skills (eye lid opening, first stand and walk, all $P<0.001)$. Time before eyes opened was $29 \pm 3$ vs. $15 \pm 1 \mathrm{~h}$ in

Table 1. Nutrition regimens $(\mathrm{ml} / \mathrm{kg} / \mathrm{d})$

\begin{tabular}{|c|c|c|c|c|c|c|c|c|}
\hline \multirow[b]{2}{*}{ Day } & \multicolumn{2}{|c|}{ Experiment 1} & \multicolumn{3}{|c|}{ Experiment 2} & \multicolumn{3}{|c|}{ Experiment 3} \\
\hline & TPN & MEN-BC & TPN & MEN-BC & MEN-IF & EN-BC & EN-IF & EN-HM \\
\hline 1 & 96 & $79+16$ & 96 & $79+16$ & $79+16$ & $96+16$ & $96+16$ & $96+16$ \\
\hline 2 & 120 & $86+32$ & 120 & $86+32$ & $86+32$ & $96+32$ & $96+32$ & $96+32$ \\
\hline 3 & 144 & $93+48$ & 144 & $93+48$ & $93+48$ & $96+48$ & $96+48$ & $96+48$ \\
\hline 4 & 144 & $76+64$ & 144 & $76+64$ & $76+64$ & $96+64$ & $96+64$ & $96+64$ \\
\hline 5 & \multicolumn{2}{|c|}{ Euthanasia-dissection } & \multicolumn{3}{|c|}{ Euthanasia-dissection } & $72+88$ & $72+88$ & $72+88$ \\
\hline 6 & - & - & - & - & - & $72+112$ & $72+112$ & $72+112$ \\
\hline $7-9$ & - & - & - & - & - & $48+156$ & $48+156$ & $48+156$ \\
\hline 10 & - & - & - & - & - & \multicolumn{2}{|c|}{ Euthanasia-dissection } & \\
\hline
\end{tabular}

In each experiment, total fluid intake is given as volume of parenteral nutrition plus volume of enteral nutrition. Total energy and fluid intake was matched among different treatment groups.

BC, bovine colostrum; EN, enteral nutrition; HM, human milk; IF, infant formula; MEN, minimal enteral nutrition; TPN, total parenteral nutrition. 
Table 2. Body weight, relative gut weights, NEC score, and NEC incidence by the end of each experiment (means \pm SEM)

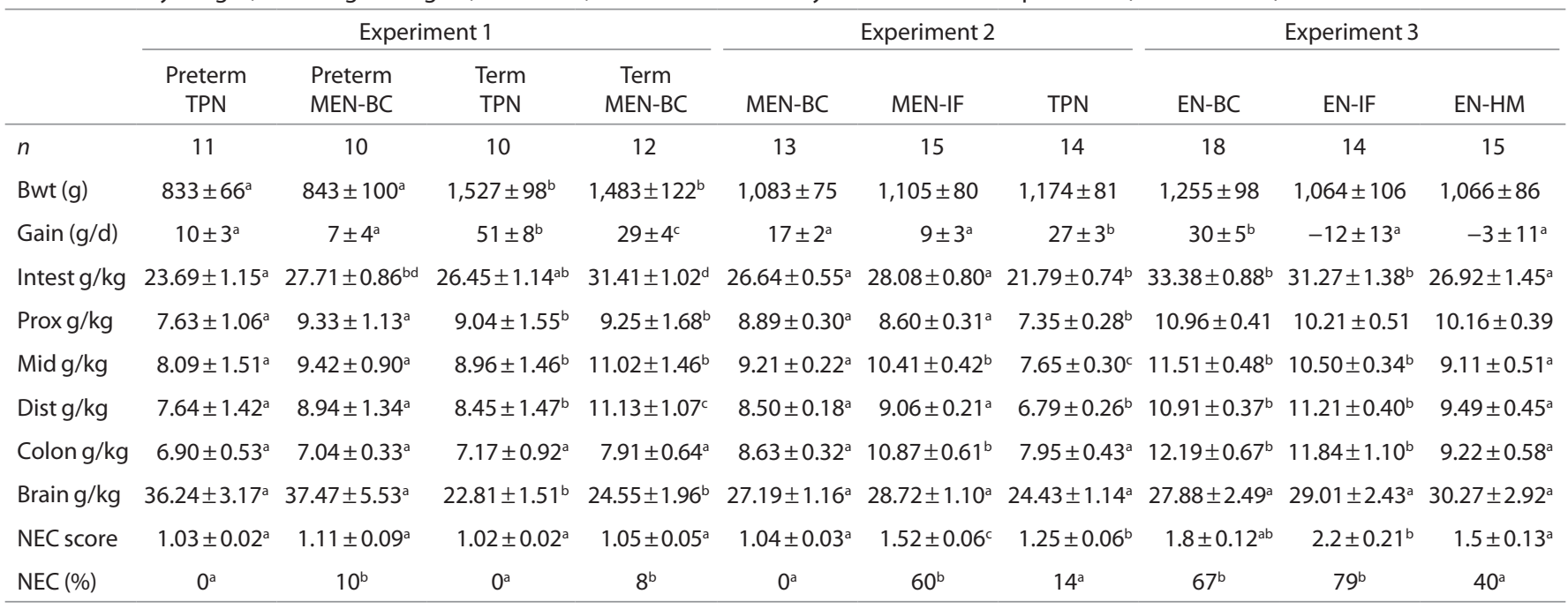

In each study, means not sharing the same superscript are significantly different $(P<0.05)$.

BC, bovine colostrum; Bwt, body weight; EN, enteral nutrition; HM, human milk; IF, infant formula; Intest, small intestine; MEN, minimal enteral nutrition; TPN, total parenteral nutrition.

preterm and term pigs, respectively, whereas time required before first stand was $26 \pm 2$ vs. $7 \pm 1 \mathrm{~h}$, and time before first walk was $31 \pm 2$ vs. $9 \pm 1 \mathrm{~h}$ in preterm and term pigs.

\section{Home Cage Activity Level}

In experiment 1 , a compromised neonatal arousal was observed in preterm pigs, relative to the term pigs, as indicated by the presence of more activity counts $(361 \pm 11$ vs. $315 \pm 14 ; P<0.05)$, but shorter length of active time $(15.5 \pm 0.6$ vs. $21.3 \pm 0.8 \% ; P<0.01)$. No difference in activity counts was observed between TPN and MEN-BC pigs $(P=0.39)$. With regard to activity counts, there was no interaction between diet and gestational age at birth, while for proportion of active time, the MEN diet showed increased values, relative to TPN, but only for term pigs $(P<0.05$; Figure $1 \mathbf{b})$.

In experiment 2 , less activity counts and less active time were observed in the TPN pigs, compared with both MEN-IF and MEN-BC pigs, which showed similar values $(P<0.05$; Figure $1 c, d)$. In experiment 3 , both the activity counts and the proportion of active time were reduced for EN-IF, relative to EN-BC pigs $(P<0.05)$, with intermediate values for HM pigs (Figure 1e,f). Activity recordings were stopped on day 6 in this experiment to avoid any confounding with development of NEC disease.

\section{Quantitation of Intestinal Serotonergic Cells}

Figure 2 shows a representative photomicrograph of a preterm pig intestine, displaying immunohistochemical staining of serotonergic cells. Regional analyses of the volume density of serotonergic cells across the three treatments in experiment 2 showed that the distal small intestine contained a significantly higher density of 5-HT enteroendocrine cells than the proximal or middle small intestine $(P=0.003)$. The piglets fed MEN levels of $\mathrm{BC}$ showed increased mean volume density across the middle and distal small intestine, relative to corresponding values in MEN-IF pigs (2.88 \pm 0.29 vs. $1.96 \pm 0.56 \%$ and
$3.68 \pm 0.44$ vs. $2.77 \pm 0.65 \%$, respectively), but none of the differences reached statistical significance (both $P>0.05$ ).

\section{Circulating Levels Serotonin}

In experiment 2, a tendency to increased platelet-free plasma serotonin level was observed for MEN-BC pigs, relative to TPN pigs $(P=0.07$; Figure $3 a)$, with intermediate values for MEN-IF pigs. For platelet-related serotonin, MEN-IF pigs had higher values than MEN-BC and TPN pigs (both $P<0.05$; Figure 3b).

\section{DISCUSSION}

Infants born preterm often show complications related to the GIT (e.g., high sensitivity to feeding intolerance and NEC) and CNS (e.g., brain damage and impaired neuromuscular control). Enteral feeding shortly after birth, even with small amounts of milk (MEN, e.g., $10-50 \mathrm{ml} / \mathrm{kg} / \mathrm{d}$ ), may stimulate GIT growth and maturation and allow a more rapid transition to full enteral feeding (e.g., $150-180 \mathrm{ml} / \mathrm{kg} / \mathrm{d}$ ). This may prevent the postnatal growth restriction that is believed to have negative neurodevelopmental consequences (10). Because the gut, enteric nervous system, and the CNS are structurally and functionally connected at many levels (e.g., neuroanatomy, endocrinology, immunology, and exposure of the brain to absorbed milk neurotropic factors), it is possible that also the early maturation of the two systems are functionally connected. These links could be particularly important in the immediate postnatal period of preterm infants when the CNS, enteric nervous system, and GIT are immature. Limitations in doing infant studies make it difficult to prove this hypothesis. In a well-recognized model of preterm infants, we now document that neonatal arousal, physical activity, and GIT growth are indeed reduced in preterm vs. term pigs, delivered and reared under identical conditions, and that MEN feeding results in increased GIT growth as well as increased physical activity. While our results do not prove functional links between the GIT and brain, they do 
a

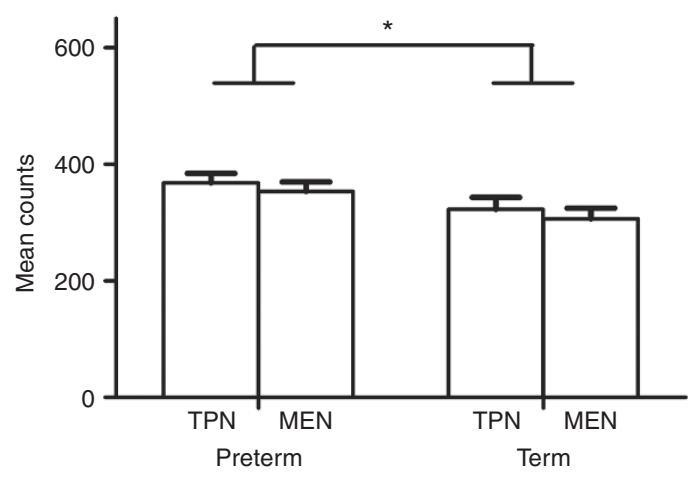

C

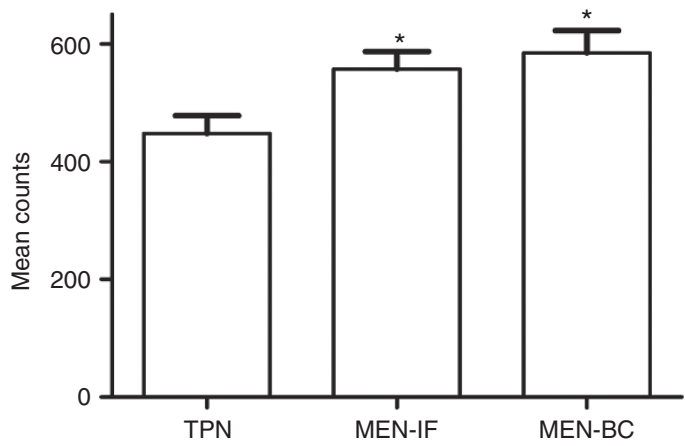

e

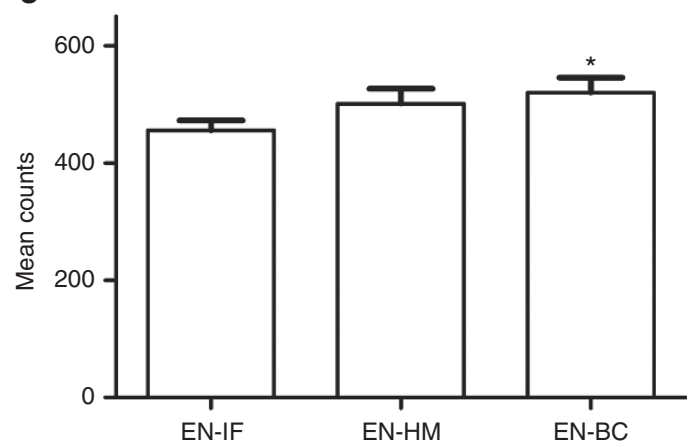

b

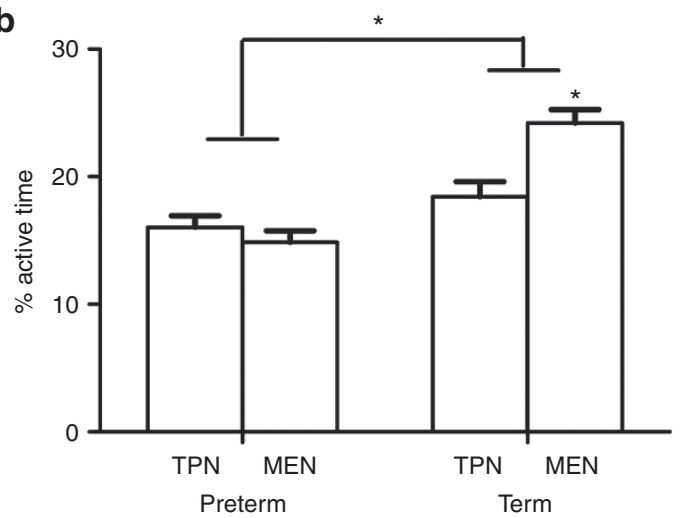

d

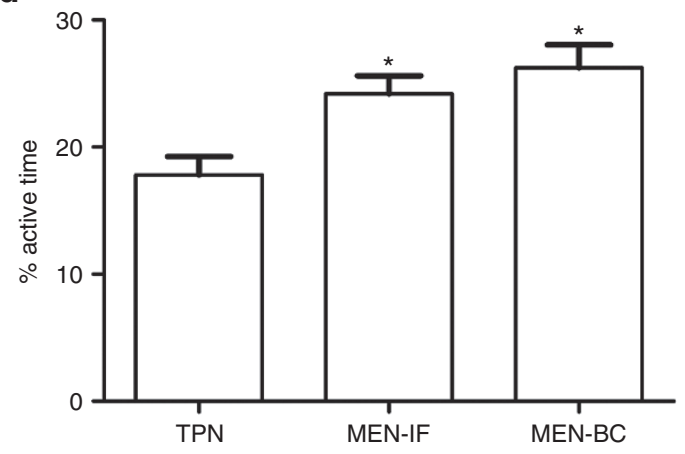

f

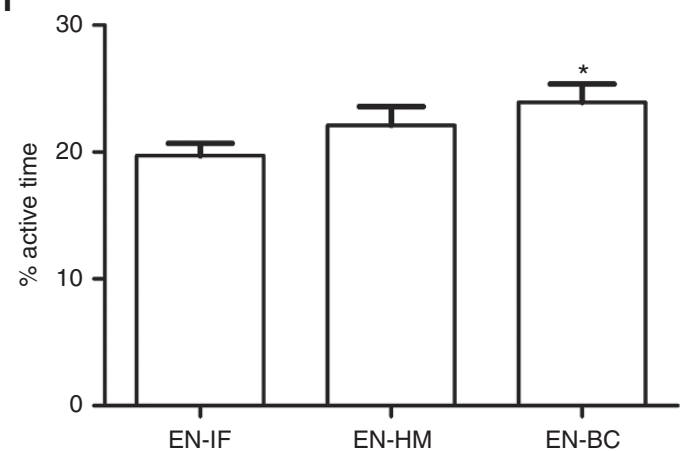

Figure 1. Activity counts (left panels) and proportion of active time (right panels) in newborn pigs (mean values $\pm S E M)$. (a,b) In experiment 1 , preterm or term pigs were fed total parenteral nutrition (TPN) or minimal enteral nutrition with bovine colostrum (MEN-BC) for $5 \mathrm{~d}$ after birth. (c,d) In experiment 2, preterm pigs were fed TPN or MEN with infant formula (MEN-IF) or bovine colostrum (MEN-BC) for $5 \mathrm{~d}\left({ }^{*} P<0.05\right.$ relative to TPN group). $(\mathbf{e}, \mathbf{f})$ In experiment 3, preterm pigs received increasing enteral nutrition with IF (EN-IF), human milk (EN-HM), or BC (EN-BC;

${ }^{*} P<0.05$ relative to EN-IF group). BC, bovine colostrum; EN, enteral nutrition; HM, human milk; IF, infant formula; MEN, minimal enteral nutrition; TPN, total parenteral nutrition.

support that stimulation of GIT maturation by enteral feeding may affect neurological functions involved in muscular control. The results indicate that neonatal stimulation of gut growth and physical activity is diet dependent, and a differential stimulation of the GIT serotonergic cells may play a role among the links between the GIT and CNS at this critical time.

When mother's own milk is not available during the first week after preterm birth, TPN, IF, or HM are commonly used to enhance nutrient and fluid intake and support GIT maturation (21). As a novel MEN supplement, BC is currently being tested in a pilot, feasibility study in preterm infants (22). As demonstrated in both infants and piglets, IF feeding is associated with an elevated risk of NEC (19), probably because of its reduced contents of milk bioactive factors. Pasteurized HM protects against NEC, relative to IF (20), but a reduced nutritional quality for preterm infants requires fortification when HM is used for longer periods (23), probably explaining that body and intestinal weights were lower in $\mathrm{HM}$ vs. BC pigs in our study. Colostrum contains high concentrations of trophic and immunoregulatory factors (24) and a rapid progression of enteral feeding with BC or porcine colostrum $(120 \mathrm{ml} /$ $\mathrm{kg} / \mathrm{d}$ from day 3 after birth) results in less NEC in preterm pigs, relative to IF $(25,26)$. It is noteworthy in this study that IF remained inferior to $\mathrm{BC}$ and $\mathrm{HM}$, even using a relatively slow 


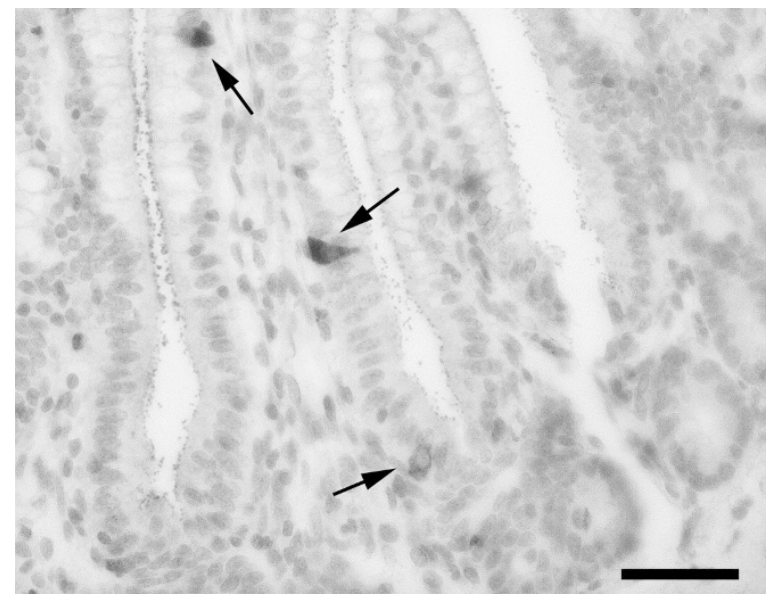

Figure 2. Representative photomicrograph of the distal intestine from a pig fed MEN-IF with serotonin-reactive cells indicated by the arrows. Bar $=100 \mu \mathrm{m}$. IF, infant formula; MEN, minimal enteral nutrition.

transition from parenteral to enteral feeding $(<64 \mathrm{ml} / \mathrm{kg} / \mathrm{d}$ for $4 \mathrm{~d}$ ). It remains unclear why even small (MEN) volumes of IF are detrimental to a newborn immature intestine. Studies in preterm pigs indicate that maltodextrin in formula (instead of lactose) may play a role and induce maldigestion and excessive nutrient fermentation (27). Furthermore, IF products are clearly inferior to intact milk products in stimulating immunity and mucosal defense mechanisms (28-30).

This study demonstrates that preterm pigs display lower physical activity level relative to term pigs over the first week of life and that a slow increase in enteral nutrition stimulates GIT growth as well as physical activity level. Preterm pigs not only showed longer time to achieve basic motor skills, but also showed a lower total proportion of active time, relative to term pigs. The lower activity level of preterm pigs is likely due to immaturity of the brain cortex that relates closely to development of motor skills. While term pigs were able to stand and walk a short distance in the home cage, preterm pigs remained lying for a longer time period after birth, although they frequently kicked their legs as part of arousal process. This explains that preterm pigs showed increased activity counts (short-lasting kicking of the legs) but decreased total proportion of active time, relative to term pigs. We also showed that IF was inferior to BC and $\mathrm{HM}$ in increasing physical activity level during the first $5 \mathrm{~d}$ after birth $(<100 \mathrm{ml} / \mathrm{kg} / \mathrm{d})$, before the feeding volumes reached the levels associated with clinical complications, feeding intolerance, and NEC which were most pronounced for the IF group $(\sim 150 \mathrm{ml} / \mathrm{kg} / \mathrm{d}$ from days $7-10$ in experiment 3 ). Despite that all pigs appeared clinically healthy during the activity recordings, we cannot exclude that part of the observed differences in physical activity between preterm and term pigs, and among the diet groups (TPN, BC, IF, HM), were indirectly caused by metabolic effects and general health state of the pigs, rather than by direct effects of maturity and diet on brain and/or neuromuscular functions.

It remains unclear how the initial enteral feeding, especially with intact milk diets like colostrum and donor milk, may stimulate physical activity. A direct neuroprotective role
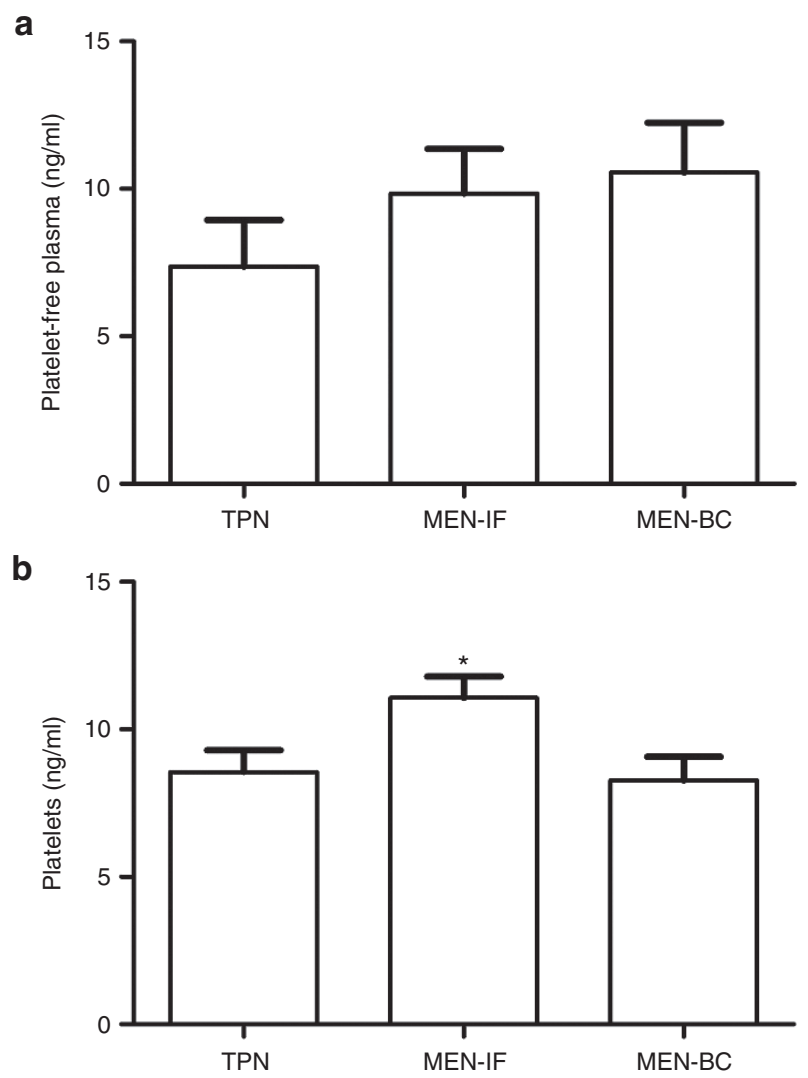

Figure 3. Blood levels of serotonin in (a) platelet-free plasma and (b) platelets for preterm pigs fed total parenteral nutrition (TPN) or minimal enteral nutrition with infant formula (MEN-IF) or bovine colostrum (MEN-BC) for 5 $\mathrm{d}$ after birth (means $\pm \mathrm{SEM} ;{ }^{*} P<0.05$ relative to TPN or MEN-BC groups). $\mathrm{BC}$, bovine colostrum; IF, infant formula; MEN, minimal enteral nutrition; TPN, total parenteral nutrition.

of colostrum is supported by the enhanced microgliogenesis and hippocampus neuron density in 3-d-old term pigs fed sow's colostrum vs. formula (28), and the colostrum-induced reduced neurological deficit in a rodent model of brain ischemia/reperfusion injury (31). As indicated above, increased physical activity in term pigs, and in MEN-fed preterm pigs, may also result from improvements in the general clinical status, secondary to enhanced nutrient absorption via improved GIT maturation. This hypothesis is supported by the higher weight gain in $\mathrm{BC}$ vs. IF pigs (experiments 2 and 3), despite that we provided identical fluid and energy to pigs across the treatments in each of the three experiments. In experiments 1 and 2, the highest weight gain was found in the TPN groups, and this likely reflects the additional body energy demand resulting from stimulation of gut growth, metabolism, and maturation by the MEN enteral nutrition. The TPN groups also tended to have lower relative brain weights than MEN pigs (although not significantly), and these results are consistent with the markedly reduced brain weights when TPN nutrition was compared with transition to full enteral feeding $(192 \mathrm{ml} / \mathrm{kg} / \mathrm{d})$ in another recent study on preterm pigs (32). Although we did not assess gut function in this study, our previous studies would suggest that age- and feeding-induced increases in relative intestinal mass in preterm pigs are closely 
associated with increases in key functions such as digestive enzyme activity, nutrient absorption, and immunological tolerance to bacterial colonization (27,33-35). Our follow-up studies show that the difference in intestinal mass per body weight between preterm and term pigs on day 5 disappears over the following weeks but that the general deficits in body growth and many behavioral characteristics (regardless of diet during the first $5 \mathrm{~d}$ ) remain until at least $4 \mathrm{wk}$ of age (36). Still, it is important to note that the conclusions of this study relate only to the first week of life.

With regard to maturity- and diet-related effects on physical activity via an increased digestive and metabolic capacity, it is important to consider emotional effects. Hence, advancing fetal age at birth and enteral feeding may allow improved release of neuropeptides from the intestine with direct effects on brain emotional centers. Related to emotional behavior, the neurotransmitter serotonin has been suggested as a central link between the gut and brain (37). Serotonin located in and secreted from the enteric nervous system regulates many aspects of intestinal function, including GIT immunity and motility (13), and the expression of GIT serotonergic cells is developmentally regulated, also in pigs (38). In our preliminary studies on the volume of intestinal serotonergic cells in experiment 2, we found an increase from middle to distal gradient in the volume density, and enteral nutrition with $\mathrm{BC}$ tended to increase the volume density of serotonergic cells distally, relative to IF and TPN. Correspondingly, the distal part of the intestine is also more sensitive to the trophic effects of enteral diet than the proximal region (see Table 2 for experiments 1 and 2), probably because of a larger number of enteroendocrine and immune cells mediating these effects (9). Our analyses of the diet-dependent secretion of serotonin also suggested that MEN-BC pigs had higher serotonin levels in platelet-free plasma, relative to TPN pigs, with intermediate values for MEN-IF pigs. Platelet-free serotonin is considered more bioactive, compared with platelets combined serotonin. Further analyses of the age-dependent secretion of serotonin and its possible passage across the blood-brain barrier, acting on CNS serotonin receptors, are required to confirm that this neurotransmitter link GIT and brain functions in early life.

In conclusion, the overall physical activity of newborn pigs depends on gestational age at birth (preterm, term), feeding mode (TPN, enteral), and diet (IF, HM, and BC). Several mechanisms may explain that IF-fed preterm pigs show impaired activity, even without any manifest clinical disease (e.g., NEC), compared with term pigs, or preterm pigs fed BC and HM. These possible mechanisms include metabolic deficits, impaired nutrient digestion, neuroanatomical immaturity, and impaired diet-dependent signals between the gut and the brain (e.g., serotonin). It is important to establish if differences in physical activity of preterm infants in early life have long-term consequences and whether a relation to gut or brain immaturities can be modulated by diet. The preterm pig may be an excellent tool to study such relations and mechanisms.

\section{METHODS}

\section{Animals and Their Treatment}

One hundred and fifty-seven preterm pigs were delivered from 10 sows by cesarean birth at 105-106 d of gestation (Large White $x$ Danish Landrace $\times$ Duroc; Askelygaard Farm, Roskilde, Denmark; term $=117 \pm 2 \mathrm{~d}$ ). The piglets were anesthetized and catheterized with orogastric feeding tubes (6-Fr, Portex; Smiths Medical, St Paul, MN), and vascular catheters (4-Fr; Portex) were inserted into an umbilical artery and reared in temperature-regulated individual incubators with oxygen supply for the first $4-6 \mathrm{~h}$. The pigs were given three doses of maternal serum (a total of $16-18 \mathrm{ml} / \mathrm{kg}$ ) during the first $24 \mathrm{~h}$ after birth for passive immunization. All pigs received PN via their vascular catheter with a solution based on a Kabiven three-chamber bag product (kindly donated by Fresenius Kabi, Bad Homburg, Germany). The PN was adjusted in nutrient composition to meet the requirements of preterm and term pigs with energy (E) as $3.210 \mathrm{~kJ} / \mathrm{l}$ and a macronutrient composition of $36 \mathrm{E} \%$ from fat (Intralipid, refined soy oil), $24 \mathrm{E} \%$ from protein, and $40 \mathrm{E} \%$ from carbohydrate, vitamins, and minerals were also added. Total daily energy intake (from both parenteral and enteral supply) was matched among different treatment groups in each experiment. The studies were approved by the National Committee on Animal Experimentation in Denmark.

Experiment 1. The preterm and term pigs were allocated into two groups according to birth weight and gender, TPN $(n=18-21)$ or PN plus BC given as MEN (MEN-BC, $n=15-19)$. The BC was a sterile powder (kindly donated by Biofiber Damino, Vejen, Denmark) produced from milk obtained from dairy cows within the first $24 \mathrm{~h}$ after parturition. The $\mathrm{BC}$ powder was diluted in bottled mineral water $(171 \mathrm{~g} / \mathrm{l})$, and daily energy intake and incremental intakes were similar for TPN and MEN-BC pigs (310-460 kJ/kg/d). PN was given continuously, whereas MEN feedings were given every $3 \mathrm{~h}$ according to the volumes shown in Table 1.

Experiment 2. The preterm pigs were allocated into three diet groups: TPN as above $(n=10)$, MEN-BC as above $(n=10)$, and MEN-IF $(n=$ 12). IF was prepared in bottled mineral water. The formula used was a commercially available product, ready to use for preterm infants (Enfalac Premature, Mead Johnson Nutrition, Glenview, IL: 165 g/l for dry matter; $810 \mathrm{kcal} / \mathrm{l}$ for energy, $24 \mathrm{~g} / \mathrm{l}$ for protein, $89 \mathrm{~g} / \mathrm{l}$ for carbohydrate including $41 \mathrm{~g} / \mathrm{l}$ for lactose and $45 \mathrm{~g} / \mathrm{l}$ for corn syrup solids, and $41 \mathrm{~g} / \mathrm{l}$ for fat). The feeding regimes are indicated in Table 1.

Experiment 3. Preterm pigs were allocated into three groups on the basis of weight and gender: PN plus BC first given as MEN (Table 1 ) and later as full enteral feeding (EN-BC, $n=18)$, PN plus HM in doses as above (Women's Milk Central, Hvidovre Hospital, Denmark; EN-HM, $n=16$ ), and PN plus a commercial preterm IF product in doses as above (Alprem, Nestlé Nutrition, Barcelona, Spain; EN-IF, $n=34$ ). For all three milk diets, the ready-to-use products were kept frozen, and the amount needed for one full day of feeding was thawed each day. Details of nutrition volume given on each day are shown in Table 1, and all three diets were diluted to have the same energy content $(\sim 3.1 \mathrm{MJ} / \mathrm{l})$.

\section{Neonatal Arousal Recordings}

For each pig in experiment 1 , the exact time when each pig was first observed with open eyes, standing, and walking were registered. Number of hours from birth to reach each of these basic motor skills was noted.

\section{Home Cage Activity Surveillance System}

Pigs were kept individually in transparent incubators with controlled ventilation and a local heat source (39). Incubators were equipped with foam pads and diapers on top of plastic grid for temperature optimization and comfort. The activity in each incubator was recorded by an infrared video surveillance camera connected to an HD recorder with built-in motion detection. The HD recorder had a digital output for each camera connected to a digital input card (DAQ card) where the status of each camera was registered as being either active or 
resting. With the PIGLWin application (Ellegaard Systems, Faaborg, Denmark), activity count (number of shifts between active/resting) and proportion of active time within each hour were automatically recorded by the HD recorder. The surveillance cameras were turned off during any handling and contact with pigs, and the activity data during the subsequent $60 \mathrm{~min}$ was excluded from the calculations. Activity surveillance ended before pigs were killed on day 5 (experiments 1 and 2), or at $9.00 \mathrm{~h}$ on day 6 (experiment 3 ). From the recordings, we excluded any activity data related to the immediate neonatal period ( $<24 \mathrm{~h}$ after birth by cesarean section). Recordings were also restricted to the period when piglets showed no clinically observable signs of NEC according to our standard criteria. Thus, recordings were continued until pigs were killed on day 5 for experiments 1 and 2 , and until day 6 in experiment 3.

\section{Clinical Evaluation and Tissue Collection}

Pigs were monitored every $3 \mathrm{~h}$ and killed if severe clinical symptoms of NEC appeared, such as abdominal distension, lethargy, cyanosis, or bloody diarrhea. Otherwise, pigs were killed (anesthesia plus sodium pentobarbitone) in the morning of day 5 (experiments 1 and 2) or on day 10 (experiment 3), as described previously (39). Body weight and wet weights of small intestine and colon were recorded. The entire small intestine was divided into three equal regions, proximal (Prox), middle (Mid), and distal (Dist), and the wet weight of each part was recorded. Three regions of small intestine, stomach, and colon were graded for pathology using a macroscopic NEC lesion score (1, no or minimal focal hyperemic gastroenterocolitis; 2 , mild focal gastroenterocolitis; 3 , moderate locally extensive gastroenterocolitis; 4 , severe focal gastroenterocolitis; 5 , severe locally extensive hemorrhagic and necrotic gastroenterocolitis; or 6, severe extensive hemorrhagic and necrotic gastroenterocolitis). Pigs with a score of 3 or more in any of the intestinal regions (Prox, Mid, Dist, or Colon) were classified as a case of NEC. Lesion severity was expressed as the means of the macroscopic inflammatory scores in the stomach, small intestine (average of Prox, Mid, and Dist), and colon.

\section{Circulating Serotonin (5-HT)}

In experiment 2, plasma samples were centrifuged at room temperature for $10 \mathrm{~min}$ at $200 \mathrm{~g}$ to obtain platelet-rich plasma (PRP). From this PRP, the platelet pellet was obtained by adding in an aliquot of $200 \mu \mathrm{l}$ PRP, $800 \mu \mathrm{l}$ of physiological saline, followed by centrifugation at $4,500 \mathrm{~g}$ for $10 \mathrm{~min}$ at $4^{\circ} \mathrm{C}$. To this pellet, $200 \mu \mathrm{l}$ of distilled water was added, and the pellet was frozen at $-20{ }^{\circ} \mathrm{C}$. After thawing, the samples were centrifuged at $10,000 \mathrm{~g}$ for $2 \mathrm{~min}$ at room temperature. The supernatant was used for estimating the serotonin content in the blood platelets. Thereafter, the PRP was centrifuged at 4,500g for $10 \mathrm{~min}$ at $4{ }^{\circ} \mathrm{C}$ to obtain the platelet-free plasma. The supernatant was used to determine the free serotonin (not bound to platelets). The serotonin concentration was determined in platelets and in plateletfree plasma by ELISA, according to the manufacturer's guidelines (IBL, Hamburg, Germany) and expressed as nanogram per milliliter.

\section{Quantitation of Intestinal Serotonergic Cells}

A subsample of the pigs from experiment $2(n=6$ from each treatment group) were used to quantify the number of serotonergic cells in different parts of the small intestine by immunohistochemical staining (38). The length of the small intestine was measured, and samples were taken at 17, 50, and $83 \%$ and designated Prox, Mid, and Dist small intestine. These full thickness tissue samples were fixated in $4 \%$ paraformaldehyde in phosphate-buffered saline $(0.01 \mathrm{~mol} / \mathrm{l}, \mathrm{pH} 7.4)$ and left to fix for $2 \mathrm{~h}$. Afterward, samples were rinsed in phosphatebuffered saline and processed for paraffin embedding. At systematically random selected positions, vertical sections were collected (4 $\mu \mathrm{m})$. After rehydration, sections were rinsed three times with Trisbuffered saline $(0.05 \mathrm{~mol} / \mathrm{l}, \mathrm{pH} 7.4)$. Endogenous peroxidase activity was depleted by incubating with $3 \% \mathrm{H}_{2} \mathrm{O}_{2}$ in Tris-buffered saline for $10 \mathrm{~min}$ at room temperature. Nonspecific staining was blocked by incubating for $1 \mathrm{~h}$ at room temperature with $20 \%$ normal swine serum diluted in Tris-buffered saline and enriched with $0.3 \%$ Triton-X-100 and $1 \%$ bovine serum albumin. Subsequently, sections were incubated overnight $\left(4{ }^{\circ} \mathrm{C}\right)$ in a humidity chamber with a polyclonal rabbit anti-5-HT antibody (1/1,000, Chemicon; Millipore, Billerica,
MA). Sections were rinsed and subsequently incubated with a biotinylated swine antirabbit antibody (1/600; Dako, Glostrup, Denmark) for $2 \mathrm{~h}$ at room temperature. After the next rinsing step, the sections were incubated with streptavidin-conjugated horseradish peroxidase $(1 / 600, D a k o)$ for $2 \mathrm{~h}$ at room temperature. After rinsing with Trisbuffered saline and distilled water, immunoreactivity was visualized by incubating the sections with the chromogen 3,3'-diaminobenzidine (Dako). The sections were counterstained with Carazzi's hematoxylin and mounted with glycerol.

An Olympus BX50 microscope connected to a computer running the software program Cast2 (Olympus, Copenhagen, Denmark) was used for the stereological analysis. One single investigator, blinded to the origin of the samples, performed the analysis. In the three different intestinal parts of the small intestine, the volume density of the cells immunoreactive (IR) for 5-HT was estimated using the following equation: $\mathrm{Vv}$ (serotonin IR cells/epithelial layer) $=\sum \mathrm{P}$ (serotonin IR cells) $/ \Sigma \mathrm{P}$ (epithelial cell layer). The number of points (of the counting grid) hitting the serotonin IR cells was referred as $\sum \mathrm{P}$ (serotonin IR cells) and $\sum \mathrm{P}$ (epithelial cell layer), referring to the number of points hitting the epithelial layer of the tunica mucosa (reference volume). The optimal density of the counting grid, the number of sections, and the number of fields were estimated using the coefficient of error of a ratio estimator (40)

\section{Statistical Analysis}

Data were analyzed by SPSS statistical software (version 13; IBM, Chicago, IL) and R (version 2.15.0; The R Foundation, Auckland, New Zealand; open source software, available online). For relative gut and brain weights, the effects of diet and gestation in experiment 1 were evaluated by a general liner model, the difference among four groups (gestation $\times$ diet) in experiment 1 and the diet effect in experiments 2 and 3 were evaluated by one-way ANOVA; post hoc comparison between groups was performed with Student-Newman-Keuls test. NEC incidence was analyzed with logistic regression analyses. In case of a perfect separation between groups, Fischer's exact test was used. Colon NEC score was analyzed using nonparametric statistics with the nparcomp function. Mean growth was calculated as difference from between $\mathrm{d} 5$ and d 1 divided by 4 and analyzed as such. Linear regression was done with multiple comparisons between groups. For activity counts and proportion of active time, the diet and gestational age effects in experiment 1 , and diet effects in experiments 2 and 3 , were evaluated by a mixed linear model. Blood levels of serotonin were evaluated by one-way ANOVA; post hoc comparison between groups was performed with Student-Newman-Keuls test. Results are presented as estimated marginal means (adjusted for litter and gender) \pm SEM. $P$ values less than 0.05 were considered statistically significant. All figures were prepared by GraphPad Prism 5 (Graph Pad Software, La Jolla, CA).

\section{STATEMENT OF FINANCIAL SUPPORT}

This research was supported by NEOMUNE Project from the Danish Research Councils and International Program 985, Sun Yat-Sen University, China.

Disclosure: University of Copenhagen has filed a patent concerning the use of bovine colostrum for pediatric patients.

\section{REFERENCES}

1. Padilla N, Alexandrou G, Blennow M, Lagercrantz H, Adén U. Brain growth gains and losses in extremely preterm infants at term. Cereb Cortex; e-pub ahead of print 31 January 2014.

2. Richardson HL, Horne RS. Arousal from sleep pathways are affected by the prone sleeping position and preterm birth: preterm birth, prone sleeping and arousal from sleep. Early Hum Dev 2013;89:705-11.

3. Zotter H, Grossauer K, Reiterer F, Pichler G, Mueller W, Urlesberger B. Is bladder voiding in sleeping preterm infants accompanied by arousals? Sleep Med 2008;9:137-41.

4. Yerushalmy-Feler A, Marom R, Peylan T, et al. Electroencephalographic characteristics in preterm infants born with intrauterine growth restriction. J Pediatr 2014;164:756-761.e1.

5. Dusing SC, Izzo TA, Thacker LR, Galloway JC. Postural complexity differs between infant born full term and preterm during the development of early behaviors. Early Hum Dev 2014;90:149-56. 
6. Månsson J, Stjernqvist K. Children born extremely preterm show significant lower cognitive, language and motor function levels compared with children born at term, as measured by the Bayley-III at 2.5 years. Acta Paediatr 2014;103:504-11.

7. Moreira RS, Magalhães LC, Alves CR. Effect of preterm birth on motor development, behavior, and school performance of school-age children: a systematic review. J Pediatr (Rio J) 2014;90:119-34.

8. Husby IM, Skranes J, Olsen A, Brubakk AM, Evensen KA. Motor skills at 23 years of age in young adults born preterm with very low birth weight. Early Hum Dev 2013;89:747-54.

9. Dockray GJ. Gastrointestinal hormones and the dialogue between gut and brain. J Physiol 2014;592(Pt 14):2927-41.

10. Embleton ND. Early nutrition and later outcomes in preterm infants. World Rev Nutr Diet 2013;106:26-32.

11. Hadders-Algra M. Prenatal and early postnatal supplementation with long-chain polyunsaturated fatty acids: neurodevelopmental considerations. Am J Clin Nutr 2011;94:Suppl 6:1874S-9S.

12. Davis-Bruno K, Tassinari MS. Essential fatty acid supplementation of DHA and ARA and effects on neurodevelopment across animal species: a review of the literature. Birth Defects Res B Dev Reprod Toxicol 2011;92:240-50.

13. Stilling RM, Dinan TG, Cryan JF. Microbial genes, brain \& behaviour - epigenetic regulation of the gut-brain axis. Genes Brain Behav 2014;13:69-86.

14. Bercik P, Park AJ, Sinclair D, et al. The anxiolytic effect of Bifidobacterium longum NCC3001 involves vagal pathways for gut-brain communication. Neurogastroenterol Motil 2011;23:1132-9.

15. Oste M, Van Ginneken CJ, Van Haver ER, Bjornvad CR, Thymann T, Sangild PT. The intestinal trophic response to enteral food is reduced in parenterally fed preterm pigs and is associated with more nitrergic neurons. J Nutr 2005;135:2657-63.

16. van Haver ER, de Vooght L, Oste M, et al. Postnatal and diet-dependent increases in enteric glial cells and VIP-containing neurones in preterm pigs. Neurogastroenterol Motil 2008;20:1070-9.

17. Leaf A, Dorling J, Kempley S, et al.; Abnormal Doppler Enteral Prescription Trial Collaborative Group. Early or delayed enteral feeding for preterm growth-restricted infants: a randomized trial. Pediatrics 2012;129: e1260-8.

18. Clark DA, Munshi UK. Feeding associated neonatal necrotizing enterocolitis (Primary NEC) is an inflammatory bowel disease. Pathophysiology 2014;21:29-34.

19. Bjornvad CR, Thymann T, Deutz NE, et al. Enteral feeding induces dietdependent mucosal dysfunction, bacterial proliferation, and necrotizing enterocolitis in preterm pigs on parenteral nutrition. Am J Physiol Gastrointest Liver Physiol 2008;295:G1092-103.

20. Jensen ML, Sangild PT, Lykke M, et al. Similar efficacy of human banked milk and bovine colostrum to decrease incidence of necrotizing enterocolitis in preterm piglets. Am J Physiol Regul Integr Comp Physiol 2013;305:R4-R12.

21. Yu VY. Enteral feeding in the preterm infant. Early Hum Dev 1999;56: 89-115.

22. Li Y, Sangild P, Shen R, et al. Bovine colostrum for preterm infants as minimal enteral nutrition: a pilot feasibility study (PreColos-NEOMUNE) (abstract). The 48th Meeting of the European Association for Pediatric Gastroenterology, Hepatology and Nutrition, Amsterdam, The Netherlands, 6-9 May 2015.
23. Arslanoglu S, Ziegler EE, Moro GE; World Association of Perinatal Medicine Working Group On Nutrition. Donor human milk in preterm infant feeding: evidence and recommendations. J Perinat Med 2010;38:347-51.

24. Chatterton DE, Nguyen DN, Bering SB, Sangild PT. Anti-inflammatory mechanisms of bioactive milk proteins in the intestine of newborns. Int J Biochem Cell Biol 2013;45:1730-47.

25. Støy AC, Heegaard PM, Thymann T, et al. Bovine colostrum improves intestinal function following formula-induced gut inflammation in preterm pigs. Clin Nutr 2014;33:322-9.

26. Møller HK, Thymann T, Fink LN, Frokiaer H, Kvistgaard AS, Sangild PT. Bovine colostrum is superior to enriched formulas in stimulating intestinal function and necrotising enterocolitis resistance in preterm pigs. Br J Nutr 2011;105:44-53.

27. Thymann T, Møller HK, Stoll B, et al. Carbohydrate maldigestion induces necrotizing enterocolitis in preterm pigs. Am J Physiol Gastrointest Liver Physiol 2009;297:G1115-25.

28. Pierzynowski S, Ushakova G, Kovalenko T, et al. Impact of colostrum and plasma immunoglobulin intake on hippocampus structure during early postnatal development in pigs. Int J Dev Neurosci 2014;35:64-71.

29. Puiman PJ, Jensen M, Stoll B, et al. Intestinal threonine utilization for protein and mucin synthesis is decreased in formula-fed preterm pigs. J Nutr 2011;141:1306-11.

30. Siggers J, Sangild PT, Jensen TK, et al. Transition from parenteral to enteral nutrition induces immediate diet-dependent gut histological and immunological responses in preterm neonates. Am J Physiol Gastrointest Liver Physiol 2011;301:G435-45.

31. Choi HS, Ko YG, Lee JS, et al. Neuroprotective effects of consuming bovine colostrum after focal brain ischemia/reperfusion injury in rat model. Nutr Res Pract 2010;4:196-202.

32. Choudhri AF, Sable HJ, Chizhikov VV, Buddington KK, Buddington RK. Parenteral nutrition compromises neurodevelopment of preterm pigs. J Nutr 2014;144:1920-7.

33. Sangild PT, Petersen YM, Schmidt M, et al. Preterm birth affects the intestinal response to parenteral and enteral nutrition in newborn pigs. J Nutr 2002;132:3786-94.

34. Sangild PT, Siggers RH, Schmidt $M$, et al. Diet- and colonizationdependent intestinal dysfunction predisposes to necrotizing enterocolitis in preterm pigs. Gastroenterology 2006;130:1776-92.

35. Jensen ML, Thymann T, Cilieborg MS, et al. Antibiotics modulate intestinal immunity and prevent necrotizing enterocolitis in preterm neonatal piglets. Am J Physiol Gastrointest Liver Physiol 2014;306:G59-71.

36. Andersen A, Munch S, Sangild P, et al. Preterm piglets display impaired physical activity and altered behaviour during the first weeks of life. Arch Dis Child 2014;99:A27.

37. Meneses A, Liy-Salmeron G. Serotonin and emotion, learning and memory. Rev Neurosci 2012;23:543-53.

38. Willemen SA, Che L, Dewilde S, et al. Enteric and serological distribution of serotonin and its precursor tryptophan in perinatal low and normal weight piglets. Animal 2014;8:792-9.

39. Sangild PT, Thymann T, Schmidt M, Stoll B, Burrin DG, Buddington RK. Invited review: the preterm pig as a model in pediatric gastroenterology. J Anim Sci 2013;91:4713-29.

40. Gundersen HJ, Jensen EB. The efficiency of systematic sampling in stereology and its prediction. J Microsc 1987;147(Pt 3):229-63. 